\title{
Large-Signal Theory of the Effect of Dispersive Propagation on the Intensity Modulation Response of Semiconductor Lasers
}

\author{
Eva Peral, Member, IEEE, and Amnon Yariv, Life Fellow, IEEE, Fellow, OSA
}

\begin{abstract}
We have derived an exact large-signal theory of propagation in a dispersive fiber of an optical wave with sinusoidal amplitude and frequency modulation. This has been applied to the study of large-signal direct-modulation of semiconductor lasers. It is shown that the large-signal response can significantly deviate from the predictions of the small-signal theory. In particular, the improvement in modulation response caused by frequency-to-intensity modulation conversion in propagation that occurs with small-signal modulation is no longer achieved with large-signal modulation, which could affect systems such as dispersion supported transmission. Experimental results confirm our theory.
\end{abstract}

Index Terms-Frequency modulation, intensity modulation, optical fiber communication, optical fiber dispersion, optical fiber measurement applications, semiconductor lasers.

\section{INTRODUCTION}

$\mathbf{T}$ HE technologically important problem of the effect of dispersive fiber propagation on the intensity modulation response of semiconductor lasers has been treated in the past by assuming either small-signal modulation [1], [2] or low dispersion [3]. In this work we extend the analysis to the case of large-signal and arbitrary dispersion, which more closely approximates conditions in intensity modulation-direct detection (IM-DD) systems. We show that the highpass behavior of the fiber transfer function that is predicted by the small-signal theory [1] is degraded as the amplitude of the modulation signal is increased. This could affect systems that use dispersion to enhance the modulation response after propagation in fiber, such as dispersion supported transmission [1], or after a fiber Bragg grating [4].

\section{THEORY}

\section{A. 1-Tone Large-Signal Modulation}

Semiconductor lasers exhibit chirp, i.e., the frequency of the optical field is modulated whenever the intensity is modulated. To a first approximation, the intensity and frequency of the light generated by a laser directly modulated at a frequency $\Omega / 2 \pi$

Manuscript received July 1, 1999. This work was supported by the Defense Advanced Research Projects Agency, the Office of Naval Research, and the Air Force Office of Scientific Research under Award N00014-91-J-1195.

E. Peral was with the Department of Applied Physics, California Institute of Technology, Pasadena, CA 91125 USA. She is now with Ortel Corporation, Alhambra, CA 91803 USA (e-mail: eperal@ortel.com).

A. Yariv is with the Department of Applied Physics, California Institute of Technology, Pasadena, CA 91125 USA.

Publisher Item Identifier S 0733-8724(00)00392-3. vary linearly with modulation (the general case is considered below). The complex electric field amplitude, $\tilde{E}$, at the laser output can be expressed in the form

$$
\begin{aligned}
& \tilde{E}(t, z=0) \\
& \quad=P_{0}^{1 / 2}\left(1+m_{\mathrm{IM}} \cos \left(\Omega t+\varphi_{\mathrm{IM}}\right)\right)^{1 / 2} e^{i m_{\mathrm{FM}} \sin \left(\Omega t+\varphi_{\mathrm{FM}}\right)}
\end{aligned}
$$

where $P_{0}$ is the average output power, $m_{\mathrm{IM}}$ and $m_{\mathrm{FM}}$ are the intensity modulation (IM) and frequency modulation (FM) indexes, respectively, and $\varphi_{\mathrm{IM}}$ and $\varphi_{\mathrm{FM}}$ are the corresponding phases. The ratio $\left(m_{\mathrm{FM}} / i m_{\mathrm{IM}}\right) e^{i \Delta \varphi}$, where $\Delta \varphi=\varphi_{\mathrm{FM}}-\varphi_{\mathrm{IM}}$, will be referred to in what follows as the phase-to-intensity (modulation index) ratio, PIR. For light produced by a semiconductor laser, the PIR is a function of the modulation frequency, and can be expressed as

$$
\mathrm{PIR} \equiv \frac{m_{\mathrm{FM}}}{i m_{\mathrm{IM}}} e^{i \Delta \varphi}=-\frac{\alpha}{2}\left(1+\frac{\kappa(\Omega)}{i \Omega}\right)
$$

where $\alpha$ is the linewidth enhancement factor, and $\kappa(\Omega)$ is related to several laser parameters and contributes to a quasi-adiabatic chirp [5], [6].

Equation (1) indicates that the electric field spectrum consists of sidebands at harmonics of the modulation frequency centered on the laser optical frequency. Due to group velocity dispersion, linear propagation in a dispersive optical fiber produces a phase change of the sidebands relative to the optical carrier, which results in partial conversion of FM into IM, which is then photodetected at the fiber output.

Small-signal analyses have been previously proposed that only take into account the fundamental sideband at the modulation frequency. This is a reasonable approximation for transmitters with no chirp, but it does not well describe semiconductor laser light, which exhibits large frequency modulation even for relatively small intensity modulation. In the analysis presented here we assume that the IM can be still linearized, but keep the exact form of the FM. Thus, the IM is approximated by an amplitude modulation (AM) as follows:

$$
\begin{aligned}
& \left(1+m_{\mathrm{IM}} \cos \left(\Omega t+\varphi_{\mathrm{IM}}\right)\right)^{1 / 2} \\
& \quad \cong m_{0}\left(1+m_{A M} \cos \left(\Omega t+\varphi_{\mathrm{IM}}\right)\right)
\end{aligned}
$$

where, as a first approximation, $m_{0} \cong 1$ and the $\mathrm{AM}$ index $m_{A M} \cong m_{\mathrm{IM}} / 2$ (see Appendix A for a more accurate expression for $m_{0}$ and $m_{A M}$ ). By using (3) and expanding the FM as 
a series of Bessel functions [7], the electric field amplitude can be written as:

$$
\begin{gathered}
\tilde{E}(t, z=0) \\
\cong P_{0}^{1 / 2}\left(1+\frac{m_{\mathrm{IM}}}{2} \cos \left(\Omega t+\varphi_{\mathrm{IM}}\right)\right) e^{i m_{\mathrm{FM}} \sin \left(\Omega t+\varphi_{\mathrm{FM}}\right)} \\
=P_{0}^{1 / 2} \sum_{n=-\infty}^{\infty} c_{n}(z=0) e^{i n\left(\Omega t+\varphi_{\mathrm{IM}}\right)} \\
c_{n}(z=0)=e^{i n \Delta \varphi}\left[J_{n}\left(m_{\mathrm{FM}}\right)+\frac{m_{\mathrm{IM}}}{4}\right. \\
\left.\cdot\left(J_{n+1}\left(m_{\mathrm{FM}}\right) e^{i \Delta \varphi}+J_{n-1}\left(m_{\mathrm{FM}}\right) e^{-i \Delta \varphi}\right)\right] .
\end{gathered}
$$

In propagation through the fiber the different harmonics acquire different phase changes due to dispersion. The harmonic amplitude at $z$ can be expressed as

$$
c_{n}(z)=e^{i n^{2} \theta(z)} c_{n}(0)
$$

where $\theta(z)=-(1 / 2) \beta_{2} \Omega^{2} z, \beta_{2}$ is the fiber dispersion parameter and $z$ is the fiber length. The detected photocurrent, $\tilde{I}_{\text {det }}(t, z)$, is obtained by squaring the electric field amplitude. In previous analyses, this was calculated either numerically [8] or by using the small-signal approximation. Here, we have applied a summation theorem of the Bessel functions [7] to derive the exact $\tilde{I}_{\mathrm{det}}$ for an electric field with AM and FM as in (4), and is given by

$$
\begin{aligned}
\tilde{I}_{\mathrm{det}}(t, z)= & \sum_{n=-\infty}^{\infty} I_{\mathrm{det}}(n \Omega, z) e^{i n\left(\Omega t+\varphi_{\mathrm{IM}}\right)} / L(z) \\
I_{\mathrm{det}}(n \Omega, z)= & R(n \Omega) P_{0} i^{n} e^{i n \Delta \varphi} \\
& \cdot\left[J_{n}(u)-i \frac{m_{\mathrm{IM}}}{2} \cos n \theta\right. \\
& \left.\left(J_{n-1}(u) e^{-i \Delta \varphi}-J_{n+1}(u) e^{i \Delta \varphi}\right)\right]
\end{aligned}
$$

where $u=2 m_{\mathrm{FM}} \sin n \theta, I_{\mathrm{det}}(n \Omega, z)$ photocurrent at the $n$th harmonic; $R(n \Omega)$ photodiode responsivity at frequency $n \Omega$; $L(z)$ fiber power loss in linear units.

The fiber modulation transfer function relates the photodetected current at the modulation frequency after and before propagation in dispersive fiber. From (8), we obtain

$$
H_{f i b}(\Omega, z)=\cos \theta\left(J_{0}(u)-J_{2}(u) e^{i 2 \Delta \varphi}\right)-2 \frac{e^{i \Delta \varphi}}{i m_{\mathrm{IM}}} J_{1}(u)
$$

which simplifies in the small-signal approximation to the wellknown expression

$$
\begin{aligned}
H_{f i b}(\Omega, z) & \approx \cos \theta-2 \frac{m_{\mathrm{FM}}}{i m_{\mathrm{IM}}} e^{i \Delta \varphi} \sin \theta \\
& =\cos \theta-2 \mathrm{PIR} \sin \theta .
\end{aligned}
$$

Equation (10) indicates that the small-signal fiber transfer function only depends on known fiber properties, namely $\beta_{2}$ and fiber length, and the PIR, which is a laser intrinsic property. In fact, by measuring the small-signal fiber transfer function for several lengths of fiber, the laser PIR can be determined as a function of modulation frequency [5]. In addition, from (8) (or (16) in Appendix A) and (9) we observe that the large-signal response of the laser after propagation in fiber is also fully characterized by the laser PIR and the laser intensity modulation index $m_{\mathrm{IM}}$.

\section{B. N-Tone Large-Signal Modulation}

The analysis presented in the previous section can be extended to the case of an input signal consisting of an arbitrary number $N$ of tones at angular frequencies $\Omega_{k}$ (and also the case where the nonlinear dynamics of the laser cannot be neglected). The complex electric field envelope can be expressed as

$$
\begin{aligned}
& \tilde{E}(t, z=0) \cong P_{0}^{1 / 2}\left(1+\sum_{k=1}^{N} \frac{m_{I M_{k}}}{2} \cos \left(\Omega_{k} t+\varphi_{I M_{k}}\right)\right) \\
& \cdot \exp \left(i \sum_{k=1}^{N} m_{F M_{k}} \sin \left(\Omega_{k} t+\varphi_{F M_{k}}\right)\right) .
\end{aligned}
$$

By using the same type of analysis as above, we obtain that the detected signal after propagation in dispersive fiber is given by

$$
\begin{aligned}
\tilde{I}_{\mathrm{det}}(t, z)= & \sum_{n_{1}, n_{2}, \cdots, n_{N}=-\infty}^{\infty} I_{\mathrm{det}}\left(\sum_{k=1}^{N} n_{k} \Omega_{k}, z\right) \\
& \cdot \exp \left(i \sum_{k=1}^{N} n_{k}\left(\Omega_{k} t+\varphi_{I M_{k}}\right)\right) / L(z) .
\end{aligned}
$$

The photodetected intensity for the intermodulation product (IMP) $\Omega_{\mathrm{IMP}} \equiv \sum_{k=1}^{N} n_{k} \Omega_{k}$ is given by

$$
\begin{aligned}
& I_{\mathrm{det}}\left(\Omega_{\mathrm{IMP}}, z\right) \\
& =R\left(\Omega_{\mathrm{IMP}}\right) P_{0} i \sum_{k=1}^{N} n_{k} \exp \left(i \sum_{k=1}^{N} n_{k} \Delta \varphi_{k}\right) \\
& \cdot \prod_{k=1}^{N}\left(J_{n_{k}}\left(u_{k}\right)\right)\left[1-\sum_{k=1}^{N} i \frac{m_{I M_{k}}}{2} \cos \theta_{k}\right. \\
& \left.\cdot\left(J_{n_{k}-1}\left(u_{k}\right) e^{-i \Delta \varphi_{k}}-J_{n_{k}+1}\left(u_{k}\right) e^{i \Delta \varphi_{k}}\right) / J_{n_{k}}\left(u_{k}\right)\right]
\end{aligned}
$$

where $u_{k}=2 m_{F M_{k}} \sin \theta_{k}$ and $\theta_{k}=-(1 / 2) \beta_{2} \Omega_{k} \Omega_{\mathrm{IMP}} z$. The total intermodulation distortion at a particular frequency is equal to the sum of all the IMP's in (12) that fall onto that frequency.

In Appendix B the theory here developed is applied to the case of a typical CATV transmission system.

\section{SimUlation}

In this section, the validity of the approximation in (3), i.e., linearization of the laser IM, is verified by comparison with the exact analysis. The laser PIR is assumed to be that of the MQW-DFB laser tested below ( $\alpha t \cong-4$ ). Fig. 1 shows the modulation transfer function after $85 \mathrm{~km}$ of standard fiber for several intensity modulation indexes. It is observed that the large-signal fiber transfer function deviates significantly 


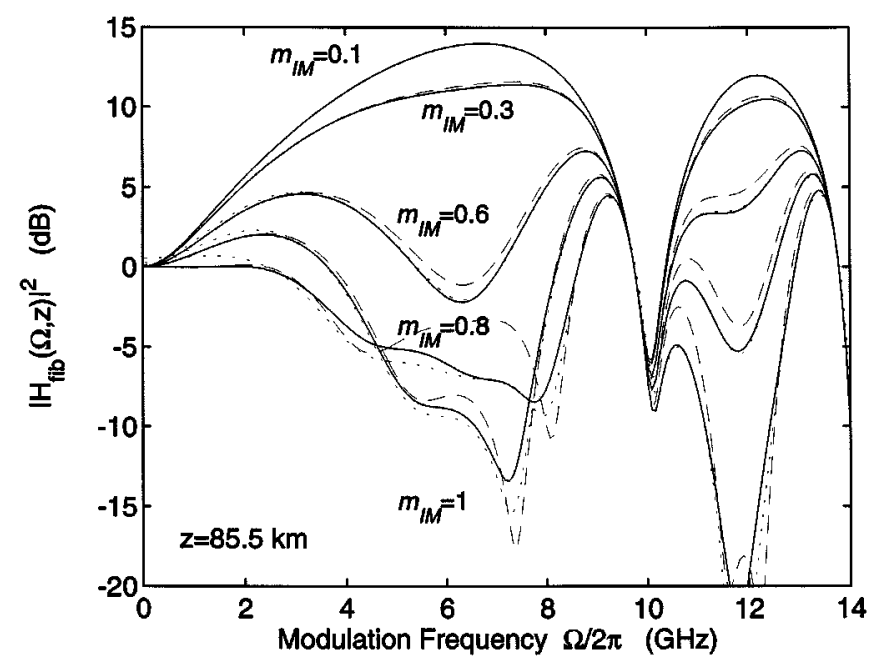

Fig. 1. Numerical simulations of fiber modulation transfer function after propagation in $85 \mathrm{~km}$ of fiber for different modulation indexes. Dashed from (9), dotted from (16) and solid is exact.

from the small-signal model for intensity modulation indexes greater than 0.2 . On the contrary, (16) remains accurate even for intensity modulation indexes close to unity. The major discrepancy occurs at the dips of the transfer function, where the received intensity is very small and the effect of the small IM sidebands becomes more important.

Comparison of (9) and (10) (as can also be observed in Fig. 1) indicates that the maximum of the fiber transfer function for any given laser parameters occurs at the smallest intensity modulation. Thus, the enhancement of modulation response due to propagation that is attained in the small-signal regime is suppressed as $m_{\mathrm{IM}}$ is increased. This could pose severe limitations to systems such as dispersion supported transmission [1] that rely on the improvement in the modulation response to combat dispersion.

\section{EXPERIMENT}

The predictions of our large-signal theory have been compared to the measured photocurrent of light from a directly-modulated MQW-DFB laser at $1.55 \mu \mathrm{m}$ after propagation in several lengths of standard fiber. The resonance frequency, the damping coefficient and the PIR were determined from the small-signal modulation response before and after propagation in fiber. The method presented in [5] allows precise determination of the PIR. Here, we used instead a simpler method that consists of fitting the small-signal transfer function to the theoretical PIR for a MQW laser [6]. This method is accurate enough and overcomes the problems encountered with other oversimplified techniques (see [5] for more details). The intensity modulation index, $m_{\mathrm{IM}}$, was determined by measuring the modulation response before propagation in fiber and subtracting the response of the receiver (photodiode + amplifier). The receiver spectral response was determined by using the amplified spontaneous emission noise of an erbium-doped fiber amplifier as white noise source. The absolute value of the receiver gain was measured by using the known spectral density of shot noise.
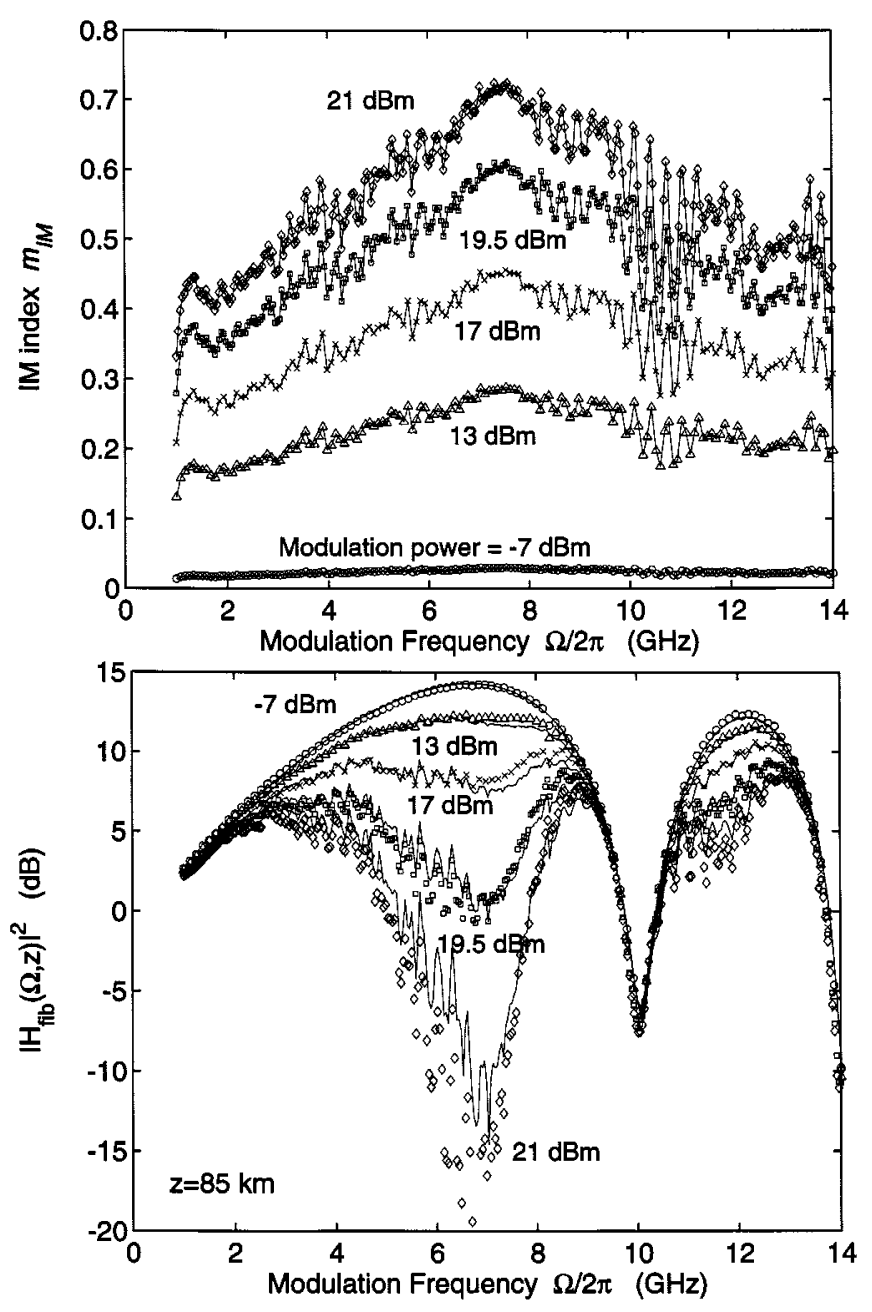

Fig. 2. (a) Intensity modulation index measured at the laser output for several modulation powers. (b) Fiber modulation transfer function after propagation in $85 \mathrm{~km}$ of fiber for the same modulation powers as in (a). Solid curve is theory.

Fig. 2(a) shows the measured intensity modulation index, $m_{\mathrm{IM}}$, as a function of modulation frequency at the laser output for several modulation powers. The frequency dependence of $m_{\mathrm{IM}}$ is due to both the laser driving electronics and the laser dynamics. This $m_{\mathrm{IM}}$ together with the PIR, measured in the small-signal regime as described above, were used to predict the large-signal fiber transfer function, $H_{f i b}(\Omega, z)$, for 85 $\mathrm{km}$ of fiber. This is plotted in Fig. 2(b) (solid) together with the experimental data points. As $m_{\mathrm{IM}}$ increases, the highpass behavior that is attained with small-signal modulation (circles) is degraded. At very large $m_{\mathrm{IM}}$, our theory does not perfectly agree with the experiment due to the presence of IM harmonics (and consequently FM harmonics) at the laser output that were neglected in (1), and not to the approximation in (3). This was verified by estimating the effect of these higher order terms in the modulation response (a complete determination of the modulation response including these terms was not possible because the relative phase between the IM harmonics was not known).

We have also measured the harmonic distortion after propagation in fiber. Fig. 3(a) shows the power in the modulation frequency, $\Omega$, and in higher order harmonics of $\Omega$ (normalized by the power in the optical carrier) in $\mathrm{dBc}$ at the laser output 

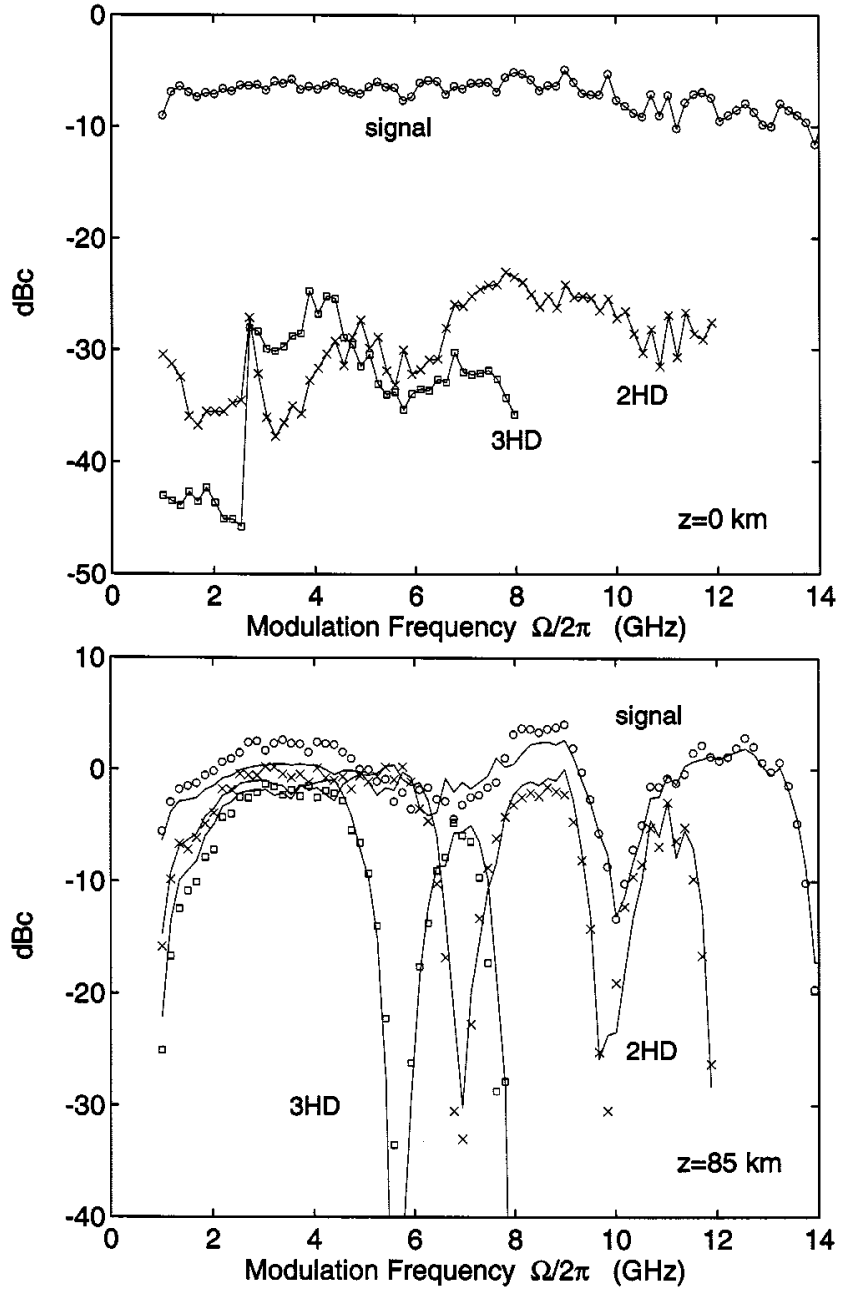

Fig. 3. Experimentally detected power in signal (circles), second harmonic (crosses) and third harmonic (squares) normalized by the power in the carrier in $\mathrm{dBc}$, (a) at the laser output, (b) after propagation in $85 \mathrm{~km}$ of fiber. Solid curve is theory.

$(z=0)$. The harmonic distortion at the laser output comes from the poor spectral purity of our signal generator, and also to a smaller degree from the nonlinearity of the laser dynamics. The measured power in the modulation tone at $\Omega$ (higher order IM harmonics were not included in the analysis) together with the PIR were used to predict harmonic distortion after propagation in $85 \mathrm{~km}$ of fiber. This theory is shown in Fig. 3(b) (solid curve), and agrees well with the experiment. Thus, we can conclude that in our case the main source of harmonic distortion after propagation comes from beating of the FM sidebands and is well described by (16). It is also observed in Fig. 3(b) that the power in the harmonics exhibits the same characteristics as that in the modulation tone, namely peaks and dips that repeat more rapidly as $\Omega$ increases and as the order of the harmonic increases.

The intermodulation distortion was measured by modulating the laser with two tones at angular frequencies $\Omega_{1}$ and $\Omega_{2}=$ $0.9 \Omega_{1}$. Fig. 4 shows the detected signal before and after propagation in fiber (in $\mathrm{dBc}$ ) at the modulation tone frequencies and in the IMP $\Omega_{2}-\Omega_{1}$, which agrees with our theoretical prediction (solid curve).

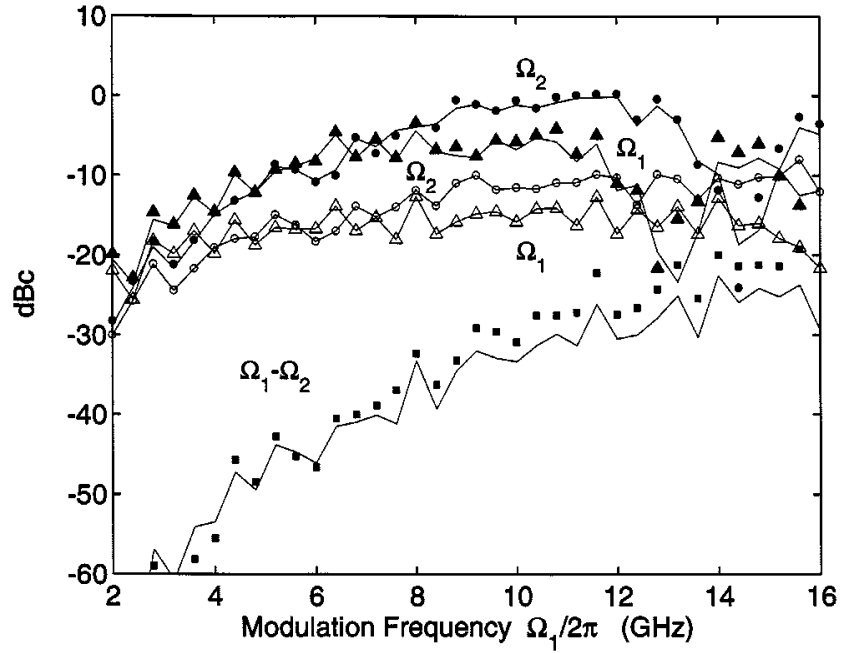

Fig. 4. Experimentally detected normalized power in $\mathrm{dBc}$ for 2-tone modulation at the laser output at $\Omega_{1}$ (open triangles) and $\Omega_{2}=0.9 \Omega_{1}$ (open circles) and after propagation in $50 \mathrm{~km}$ of fiber at $\Omega_{1}$ (solid triangles), $\Omega_{2}$ (solid triangles) and $\Omega_{1}-\Omega_{2}$ (solid squares). Solid curve is theory.

\section{CONCLUSION}

The exact large-signal theory of dispersive propagation of an optical wave with sinusoidal amplitude and frequency modulation was derived and applied to the study of large-signal direct-modulation of semiconductor lasers. Thus, an expression for the intermodulation distortion after fiber propagation for modulation with an arbitrary number of input tones was obtained. This expression fully characterizes transmission of light produced by semiconductor lasers in dispersive (linear) fiber in the frequency domain. In fact, the nonlinear transfer functions (or Volterra kernels) that describe the transmission can be derived from it straightforwardly. This analysis could be applied to the design of pre- and/or postdistortion circuits or carrier phase management schemes in SCM systems, for example to reduce intermodulation distortion. The same type of analysis as presented here has also been used to characterize dispersive propagation of light from externally frequency and/or amplitude modulated lasers, and will be subject of a future publication.

\section{APPENDIX A}

\section{A More Accurate AND AN EXaCt LARGe-Signal TheORY}

In (4) the AM index was approximated by $m_{\mathrm{IM}} / 2$. We can instead expand the IM, i.e., the left-hand side of (3), as a Fourier series

$$
\left(1+m_{\mathrm{IM}} \cos \left(\Omega t+\varphi_{\mathrm{IM}}\right)\right)^{1 / 2} \cong \sum_{l} m_{l} e^{i l \Omega t}
$$

where the Fourier coefficients, $m_{l}$, are given by [7]

$m_{l}=(-1)^{l+1} \sum_{n=0}^{\infty} \frac{(4 n+2 l-3) ! !}{(4 n+2 l) ! !}\left(\begin{array}{c}2 n+l \\ n\end{array}\right)\left(\frac{m_{\mathrm{IM}}}{2}\right)^{2 n+l}$

By keeping only the AM sideband at the modulation frequency, i.e., by using the approximation (3) with $m_{A M}=2 m_{1}$, we 
obtain that the detected photocurrent at frequency $n \omega$ is given by

$$
\begin{aligned}
I_{\text {det }}(n \Omega, z)= & R(n \Omega) P_{0} i^{n} e^{i n \Delta \varphi} \\
& \cdot\left[m_{0}^{2} J_{n}(u)+2 i m_{0} m_{1} \cos n \theta\right. \\
& \cdot\left(J_{n+1}(u) e^{i \Delta \varphi}-J_{n-1}(u) e^{-i \Delta \varphi}\right) \\
& +4 m_{1}^{2}\left(2 J_{n}(u) \cos 2 n \theta-J_{n-2}(u) e^{-i 2 \Delta \varphi}\right. \\
& \left.\left.-J_{n+2}(u) e^{i 2 \Delta \varphi}\right)\right], \quad n \geq 1 .
\end{aligned}
$$

In Section III, we show that the above expression is very accurate even for intensity modulation indexes close to unity.

If all the harmonic sidebands of the IM in (14) are taken into account, we obtain

$$
\begin{aligned}
& I_{\mathrm{det}}(n \Omega, z)=R(n \Omega) P_{0} \sum_{l, l^{\prime}=-\infty}^{\infty} i^{n+l-l^{\prime}} m_{l} m_{l^{\prime}} \\
& \cdot e^{i\left(n+l-l^{\prime}\right) \Delta \varphi} e^{i n \theta\left(l+l^{\prime}\right)} J_{n+l-l^{\prime}}(u) \text {. }
\end{aligned}
$$

Equation (17) is exact for an electric field with IM and FM. The double infinite sum in (17) converges fast and only a few terms are needed. This is simpler than previous formulations [8] that involved four infinite sums with slower convergence.

\section{APPENDIX B}

\section{INTERMODULATION DISTORTION IN CATV SYSTEMS}

As an example of the theory developed in this paper, we consider transmission of $N$ NTSC channels of CATV. At the typical CATV frequencies, the laser chirp is mostly adiabatic, and can be approximated by

$$
\mathrm{PIR} \cong-\frac{\alpha}{2} \frac{\kappa_{0}}{i \Omega}
$$

For moderate propagation distances

$$
u_{k} \cong 2 m_{F M_{k}} \theta_{k} \cong \frac{1}{2} m_{I M_{k}} \alpha \kappa_{0} \beta_{2} z \Omega_{\mathrm{IMP}}=u_{k}\left(\Omega_{\mathrm{IMP}}, z\right) .
$$

Thus, the value of the IMP's depends only on the frequency of the intermodulation product, $\omega_{\text {IMP }}$, but not on the frequency of the beating tones.

In CATV systems, small intensity modulation indexes are usually employed in order to avoid clipping of the signal. By substituting (19) into (13), and using a power series expansion of the Bessel functions, we obtain

$$
\begin{aligned}
I_{\mathrm{det}}\left(\Omega_{\mathrm{IMP}}, z\right) \cong R\left(\Omega_{\mathrm{IMP}}\right) P_{0} \prod_{k=1}^{N}\left(\frac{m_{I M_{k}}^{\left|n_{k}\right|}}{\left|n_{k}\right| !}\right) \\
\cdot\left(i \Omega_{\mathrm{IMP} \xi}\right)^{n}\left(1+\frac{n}{2 i \Omega_{\mathrm{IMP}} \xi}\right)
\end{aligned}
$$

where $\xi=(1 / 4) \alpha \kappa_{0} \beta_{2} z$ and $n=\sum_{k=1}^{N}\left|n_{k}\right|$ is the order of the IMP.
Assuming that all channels have the same intensity modulation index, $m_{I M_{k}}=m_{\mathrm{IM}}$, the $n$ th-order intermodulation distortion $\left(\mathrm{IMD}_{n}\right)$ normalized by the power in the RF carrier is approximately given by

$$
\operatorname{IMD}_{n}(\Omega, z) \approx \frac{A_{n}(\Omega)}{(n-1) !}\left(m_{\mathrm{IM}} i \Omega \xi\right)^{n-1}
$$

where

$$
A_{n}(\Omega)=\sum_{\substack{i_{1}, \cdots, i_{n}=1 \\ \Omega= \pm \Omega_{i_{1}} \pm \cdots \pm \Omega_{i_{n}}}}^{N} e^{i\left( \pm \varphi_{\mathrm{IM}_{i_{1}}} \pm \cdots \pm \varphi_{\mathrm{IM}_{i_{n}}}\right)} .
$$

From (21) and (22), it becomes evident that by proper management of the carrier phases it should be possible to affect the intermodulation distortion spectrum.

For uncorrelated carriers the total intermodulation distortion IMD is

$$
\operatorname{IMD}(\Omega, z) \approx \sum_{n} N_{n}(\Omega)\left|\frac{\left(m_{\mathrm{IM}} \Omega \xi\right)^{n-1}}{(n-1) !}\right|^{2}
$$

where $N_{n}(\Omega)=\left\langle\left|A_{n}\right|^{2}\right\rangle$ is the number of beats for that order.

\section{ACKNOWLEDGMENT}

The authors are grateful to Ortel Corporation, in particular to J. Ianelli and T. R. Chen, for providing the lasers and photodetector used in this work.

\section{REFERENCES}

[1] B. Wedding, "Analysis of fiber transfer function and determination of receiver frequency response for dispersion supported transmission," Electron. Lett., vol. 30, no. 1, pp. 58-59, Jan. 1994.

[2] A. R. Chraplyvy, R. W. Tkach, L. L. Buhl, and R. C. Alferness, "Phase modulation to amplitude modulation conversion of cw laser light in optical fibers," Electron. Lett., vol. 22, no. 8, pp. 409-411, Apr. 1986.

[3] K. Petermann and J. Wang, "Large signal analysis of FM-AM conversion in dispersive optical fibers and its application to PCM systems," Electron. Lett., vol. 27, no. 25, pp. 2347-2348, Dec. 1991.

[4] M. McAdams, E. Peral, D. Provenzano, W. K. Marshall, and A. Yariv, "Improved laser modulation response by frequency modulation to amplitude modulation conversion in transmission through a fiber grating," Appl. Phys. Lett., vol. 71, no. 7, pp. 879-881, Aug. 1997.

[5] E. Peral, W. K. Marshall, and A. Yariv, "Precise measurement of semiconductor laser chirp using effect of propagation in dispersive fiber and application to simulation of transmission through fiber gratings," J. Lightwave Technol., Oct. 1998.

[6] E. Peral and A. Yariv, "Measurement and characterization of laser chirp of multiquantum-well distributed-feedback lasers," IEEE Photon. Technol. Lett., vol. 11, pp. 307-309, Mar. 1999.

[7] M. Abramowitz and I. Stegun, Handbook of Mathematical Functions: With Formulas, Graphs, and Mathematical Tables. New York: Dover, 1976.

[8] G. J. Meslener, "Chromatic dispersion induced distortion of modulated monochromatic light employing direct detection," IEEE Quantum Electron., vol. QE-20, pp. 1208-1216, Oct. 1984. 
Eva Peral (M'98) was born on November 23, 1972, in Granada, Spain. She received the degree of Ingeniero Superior de Telecomunicaciones with Honors from the Universidad Politécnica de Valencia (UPV), Spain, in 1995, the M.S degree in electrical engineering from the California Institute of Technology (Caltech), Pasadena, in 1998, the Ph.D. degree in Ingeniería de Telecomunicaciones from UPV in 1998, and the Ph.D. degree in electrical engineering from Caltech in 1999.

During 1995, she was a Visiting Student with the Optoelectronics Group at the University of Strathclyde, Scotland, U.K., where she was engaged in integrated optics. She has worked for Philips (Spain) and Mercedes-Benz (Germany). She started her doctoral studies with the Optical Communications Group in UPV and continued in the Applied Physics Department of Caltech. She is currently working at Ortel Corporation, Alhambra, CA, as a Scientist. Her research interests include semiconductor lasers, fiber gratings, linear and nonlinear propagation in optical fiber, and integrated optics.

Dr. Peral received the First National Award for Academic Excellency from the Spanish Ministry of Culture and Education in 1996 and the IEEE/LEOS Student Fellowship Award in 1999.
Amnon Yariv (S'56-M'59-F'70-LF'95) was born in Tel Aviv, Israel. He received the B.S., M.S., and Ph.D. degrees in electrical engineering from the University of California, Berkeley, in 1954, 1956, and 1958, respectively.

After graduating from Geulah High School, Tel Aviv, he served in the first artillery unit of the Israeli Defense Forces during the 1948-1950 war of independence. In 1959, he joined Bell Laboratories, Murray Hill, NJ, where he worked on the early stages of the laser effort. In 1964, he joined the California Institute of Technology (Caltech), Pasadena, CA, as an Associate Professor of Electrical Engineering and became a Professor in 1966. In 1980, he became a Thomas G. Myers Professor of Electrical Engineering and in 1996 he became a Martin and Eileen Summerfield Professor of Applied Physics. He has taken part (along with various co-workers) in the discovery of a number of early solid-state laser systems in proposing and demonstrating the field of semiconductor integrated optics, the invention of the semiconductor distributed feedback lasers and in pioneering the field of phase conjugate optics. His present research efforts are in the areas of nonlinear optics, semiconductor lasers, and integrated optics with emphasis on communication and computation. He has published widely in the laser and optics fields and has written a number of basic texts in quantum electronics, optics, and quantum mechanics. He is Founder and Chairman of Ortel Corporation, Alhambra, CA.

Dr. Yariv is a member of the American Physical Society, Phi Beta Kappa, the American Academies of Arts and Sciences, the National Academies of Engineering and Sciences, and a Fellow of the Optical Society of America (OSA). He was the recipient of the 1980 Quantum Electronics Award of the IEEE, the 1985 University of Pennsylvania Pender Award, the 1986 Optical Society of America Ives Medal, and the 1992 Harvey Prize. 